\title{
ACQUISITION AND EXTINCTION OF VERBAL CONDITIONED RESPONSES IN MENTAL DEFECTIVES.
}

\author{
J.P. DAS \\ Utkal University, Cuttack, India.
}

In a recent article O'Connor \& Hermelin (1959) have reemphasized the role of language in learning in imbeciles. It is generally recognized that verbal conditioning may have several features not obtained in classical conditioning because of the role of verbal mediation in this form of learning (cf. Luria 1959). Therefore it is likely that mental defectives would show variations in their ability to acquire and extinguish verbal CRs corresponding to their grades of deficiency.

Many mental defectives can use verbal concepts in learning to solve simple problems. Hermelin \& O'Connor (1958) showed that imbeciles too are capable of using verbal concepts. But how simple must the concept be in order to be used by idiots or how complex can they be for morons? One of the aims of the present study is to obtain an answer to those questions.

The other aspect of this study concerns the ability of mental defectives in reversal learning. In the paper already cited, the authors studied reversal learning in imbeciles. Although they did not find any significant difference between normals and imbeciles in the acquisition of a discriminatory learning response, it cannot be inferred that there would be no difference in the case of verbal conditioning. Following Luria, verbal conditioning tasks have been designed and used where acquisition, extinction and reacquisition of a verbal-motor habit is studied (O'Connor \& Das 1959). The rationale for these reversal learning experiments is provided by the pioneering investigations of Pavlov which aimed at studying the lability or inertness of excitatory and inhibitory processes. (Pavlov 1928, 1941). Luria has noticed some peculiarities in the responses of mental defectives in such verbal conditioning experiments. But problems like the relationship between grades of mental deficiency and ability to acquire or extinguish verbal CRs do not seem to have been specifically studied. Even if mental defectives differ in these abilities, we do not know whether they are more or less 'labile' according to their intelligence level.

\section{METHOD}

Tasks The tasks were three forms of verbal conditioning. $S$ was required either to press or not to press a key at the appearance of a certain signal. The signals were given from a box-like apparatus having three patterns of lights. Task 1 consisted of pressing the key when the middle light (a single white electric bulb) was on. Task 2 consisted of pressing the key when a pattern of 4 lights (yellow, violet, red and green) on the right side were on and not to press when the same set of lights on the left side were on. In task $S$ was required to discriminate between two patterns of lights on the right side differing only in one light. The patterns were yellow, violet, red and green, and yellow, violet, pink and green. For both the patterns the same yellow, violet and green lights were on. Only red and pink distinguished the patterns. It was thought a prior that the tasks were hierarchically related in difficulty level. In each task, after $S$ had acquired the habit, he was made to extinguish it by being requir- 
ed to do the opposite and after successful extinction, was required to reacquire the first habit. Thus in Task 1 , after $S$ learnt to press when the single light was on, he was told "No, don't press" as soon as he pressed in the extinction period. Again, when this was established, he was told "press" when the light appeared.

Ordinarily an $S$ was given 10 acquisition trials (C1), 20 extinction trials (Ex) and 10 reacquisition trials (C2). The criteria for learning were 5 consecutive correct responses in C1, 10 in Ex and 5 in C2. If $S$ failed to reach the criterion in $\mathrm{C} 1$ he was given a maximum number of 20 more trials to reach it and if he still failed, he was verbally instructed to give the appropriate response and $E$ ascertained that $S$ has understood what he was required to do before resuming the $\mathrm{C} 1$ session. As soon as $S$ reached the criterion, whether before or after instruction, session $\mathrm{E}$ began without any break. Similarly, if the criterion was not reached in the course of 20 trials another 10 trials were given at the end of which $S$ was instructed and then as before, $\mathrm{C} 2$ session started without any break. The procedure here was the same as for $\mathrm{C} 1$. The tasks were given in the same order to every $S$, the order being Task 1, Task 2, Task 3. After the completion of a task, $S$ was allowed about 10 mins. rest.

Reinforcement in the tasks was both verbal and material. Each correct response was followed by "good" and at the beginning and end of each session $S$ was given sweets.

Subjects The subjects were 50 mental defects ${ }^{\star}, 22$ of them were feeble-minded, 18 imbeciles and 10 idiots. Their mean age and age ranges were 17 years 1 month and $10-30$ years for feeble-minded 19 years 6 months and 11-26 years for imbeciles, and 23 years 3 months and 15-43 years for idiots. $S$ 's reported age is not necessarily his real age. Many of the patients were assigned a probable age at the time of admission to the parent institution from which they have been transferred to their present home. A few of the $S s$ were private patients.

Eighteen out of the $50 \mathrm{Ss}$ were females, 10 of them in the moron group, 5 among the imbeciles and 3 among the idiots.

\section{Results}

Success in meeting the criterion in acquisition or extinction was scored ' 1 ' and failure 'zero'. These were then transformed into proportion of ' 1 ' responses per group. Thus proportion scores for the 3 groups of mental defects in each phase of the task were available. The scores presented in Table 1 , are 20 times the actual proportion scores. Reacquisition (C2) scores except those for Task 1 were found to be very unstable. Most of the $S \mathrm{~s}$ in the lower grades were unable to give stable CRs in the C2 situation of tasks 2 and 3 because some of them appeared to be indifferent to the stimuli and some others were highly distractible. For task 1, the lower grades responded in the same way as the morons in C2 as in C1, or Ex. Analysis of variance - design without replication (Edwards 1950) was applied to the scores in Table 1 and the results are presented in Table 2. C2 scores have been omitted because these could not be obtained for most of the imbeciles and idiots.

Table 1. Acquisition and Extinction in Three Verbal Conditioning Tasks.

\begin{tabular}{l|cc|cc|cc}
\hline \multirow{2}{*}{ Groups } & \multicolumn{7}{|c}{ Tasks. } \\
\cline { 2 - 8 } & \multicolumn{7}{|c|}{1} & \multicolumn{2}{c}{2} & \multicolumn{2}{c}{3} \\
\cline { 2 - 8 } & Acq. & Ext. & Acq. & Ext. & Acq. & Ext. \\
\hline Feeble-minded & 19.29 & 19.29 & 13.39 & 14.11 & 4.11 & 9.64 \\
Imbeciles & 20.00 & 19.23 & 8.61 & 6.31 & 0 & 4.31 \\
Idiots & 18.00 & 16.00 & 0 & 0 & 0 & 0 \\
\hline
\end{tabular}

Two of the main effects, groups and tasks and only one interaction, groups $\times$ tasks are significant. These results imply that (a) the feebleminded, imbeciles and idiots differ in

* Ss were patients in Bodhi Peet, Calcutta. The author is thankful to Dr. D. Ganguli and Miss R. Chose for facilities and permission given for testing the patients. 
Table 2. Results of Analysis of Variance of Scores in Table 1.

\begin{tabular}{|c|c|c|c|}
\hline Source & $\mathrm{df}$ & SS & MS \\
\hline Groups (G) & 2 & 175.298 & $87.649 \star \star$ \\
\hline Tasks (T) & 2 & 788.747 & $394.373 * \star$ \\
\hline Acq/Ext. (AE) & 1 & 1.674 & 1.674 \\
\hline $\mathrm{G} \times \mathrm{T}$ & 4 & 71.804 & 17.951 * \\
\hline $\mathrm{G} \times \mathrm{AE}$ & 2 & 5.759 & 2.879 \\
\hline $\mathrm{T} \times \mathrm{AE}$ & 2 & 16.159 & 8.079 \\
\hline $\mathrm{G} \times \mathrm{T} \times \mathrm{AE}$ & 4 & 6.188 & 1.549 \\
\hline Total & 17 & 1065.629 & \\
\hline
\end{tabular}

their abilities to acquire and extinguish verbal CRs, (b) the three tasks are actually different in their difficulty levels, (c) as the task difficulty increases, the defective groups show greater differences and (d) taken as a whole, mental defectives do not show significant difference between acquiring and extinguishing verbal CRs. A different kind of scores - presence or absence of spontaneous responses was also obtained for each $S$. Spontaneous response refers to a kind of response during reversals - from acquisition (C1) to extinction (Ex) and from Ex to reacquisition (C2). To illustrate, suppose in Task 2, pressing when the lights on the right side (RL) appear is correct and pressing when the lights on the left side (LL) appear is incorrect. Then during the extinction phase $(E x)$, pressing for RL must be incorrect. Usually, in the beginning of Ex trials, $\mathrm{RL}$ is presented 4 times consecutively after which RL \& LL occur randomly. At the end of these 4 consecutive RL trials, when LL is presented for the first time, an $S$ may respond to it appropriately by pressing the key. He would then be scored as showing spontaneous response. Likewise if in C2 situation, the first RL after the three LL trials is responded to correctly, he would have another spontaneous response score. Tables 2 and 3 alone gave opportunity for spontaneous responses. The percentages of spontaneous responses among feeble-minded and imbeciles are presented in Table 3 . These results show that a greater percentage of spontaneous responses were given by morons as compared to imbeciles. Idiots gave no spontaneous responses.

Table 3. Percentage of Spontaneous Response.

\begin{tabular}{l|lll|lrr}
\hline \multirow{2}{*}{} & \multicolumn{5}{|c}{ Tasks } \\
\cline { 2 - 6 } & Ex & II & C2 & Ex & III \\
\hline Feeble-minded & 9.10 & 36.40 & 22.78 & 27.30 \\
Imbeciles & 0 & 22.2 & 11.10 & 11.10 \\
\hline
\end{tabular}

\section{Discussion}

It appears from the results that level of intelligence is related to the abilities of forming and extinguishing verbal CRs. However, it cannot be generalized to ranges of normal intelligence. Another measure of verbal CRs, the spontaneous responses is also found 
to be related to grades of mental deficiency. These results are comparable to similar findings on a sample of college students. The present author carried out a study where two kinds of verbal conditioning tasks were given to more than 50 college students. One of the tasks was task 3 of the present study. A significant Chi Square of 4.13 ( $\mathrm{P}<.05)$ was obtained between intelligence scores on progressive matrices and presence or absence of spontaneous response. But intelligence was not found to be significantly related to acquisition and extinction scores in task 3. However, a significant $(\mathbf{P}<.01)$ biserial correlation of .739 was obtained between intelligence and acquisition scores in the other verbal conditioning task. Perhaps it may be suggested that task 3 is not complex enough to discriminate levels of intelligence in college students.

Reversals were neither easier nor more difficult for the subjects of the present study as inferred from the insignificant variance due to acquisition/extinction. This seems to be contrary to findings reported by O'Connor and Hermelin (1959) whose imbecile Ss took for reversal learning less than one third of the trials they required for acquisition.* It is possible that since only presence or absence of conditioning (C1) extinction (Ex) and reconditioning (C2) were scored, such a difference might have been ignored. Therefore, the scores of our morons were further analyzed and the trials an $S$ took to reach the criterion in $\mathrm{C} 1$ and Ex were compared. One would expect, following O'Connor \& Hermelin that a greater number of $S$ s would take fewer trials in Ex than in C1. But excluding the failure cases and equality cases, number of $S$ s taking fewer trials was exactly the same as those taking more trials for extinction. The verbal behavior of $S$ s during testing was similar to that observed by Luria (O'Connor, 1958). Although a subject would repeat the appropriate instruction to the light signal, he might not act appropriately. Thus many Ss were observed to be repeating "no, don't press" when the lights on the left side were on during acquisition in task 2 , but none the less, would press the key inappropriately and appear quite unconcerned about their mistake. In such cases language did not serve as a regulating and directing force and a dissociation between the verbal and motor processes was in evidence. However in the simple task, task $1, S$ s would repeat to themselves "no, don't press" or "let it go on" during the extinction phase when the light was on and prevent themselves from pressing the key. It appears, then, that the lack of connection between the direct and verbal systems observed here may be a function of the complexity of the task. In quite a different study the author obtained clear evidence of such a dissociation. There, $S$ s were asked to obtain the maximum number of hits by guessing whether a 'Yes' or 'No' would be uttered when 'Yes' occurred $75 \%$ of the times. Many Ss verbalised the correct principle that if 'Yes' was guessed on every trial, the hits would be maximized

\footnotetext{
* In a personal communication $O^{\prime}$ Connor explains the discrepancy as follows. The best reason I can think of at the moment is that in analyzing your results you may be capitalizing on the relatively larger number of feeble minded subjects who might have verbal habits well developed. As we dealt with imbeciles, our analysis of course was more straightforward. There is also the difference between your technique and the one Mrs. Hermelin and I used; where you sometimes used conditioning and sometimes instruction ..., all of Mrs. Hermelin's subjects were conditioned."

The author is indebted to Dr. N. O'Connor of Institute of Psychiatry, London, for his valuable suggestions and comments.
} 
but continued to give 'no' responses (Das, 1961). Perhaps both of these findings can be explained by one and the same principle.

\section{SUMMARY}

Morons, imbeciles and idiots were tested for acquisition, extinction and reacquisition of three kinds of verbal CRs differing in their difficulty levels. The tasks were pressing or not pressing a key to light signals. An analysis of variance yielded significant effects due to grades of deficiency and tasks as well as due to grades $\times$ task interaction. It was concluded from the results that intelligence levels found among defectives are related to the ability to acquire and extinguish verbal CRs and as the tasks become more complex, this difference in ability between morons, imbeciles and idiots increases. Some implications of the observed dissociation between the motor and verbal responses were discussed in the light of Luria's work.

\section{REFERENCES}

1. Das, J. P. Mathematical solution in the acquisition of verbal CRs. J. exp. Psychol, 1961, 61, 376-378.

2. Edwards, A. L. Experimental design in psychological research New York: Rinehart, 1950.

3. Hermelin, B \& O'Connor, N. The rote and concept learning of imbeciles $J$. ment. Def. Res., 1958, 2, 21-27.

4. Luria, A.R. \& Vinogradova, O.S. An objective investigation of the dynamics of semantic systems. Brit. J. Psychol., 1959, 50, 89-105.

5. O'Connor, N. Learining and mental defect. In
Clarke, A. M. \& Clarke, A.D.B. (Eds.) Mental deficiency: The changing outlook. London: Methuen, 1958.

6. O'Connor, N., \& Das, J.P. Lability in schizophrenia Brit. J. Psychol, 1955, 50, 333-337.

7. O'Connor, N. \& Hermelin. B. Discrimination and reversal learning in imbeciles. J. abnorm. soc. Psychol., 1959, 59, 409-413.

8. Pavlov, I.P. Lectures on conditioned reflexes. Vol. I, 1928, Vol. II 1941, New York: International Publishers.

MS. received, VII 10, 61 .

J. P. Das (1931- ) 1953 M.A. Patna Univ., India; Ph.D. from Institute of Psychiatry, University of London, under Prof. Eysenck's supervision, in 1957. Research area: Hypnosis, classical and verbal conditioning, probability learning. At present, Reader in postgraduate Dept. of Psychology, Utkal Univ., Cuttack, India.

\section{NEWS OF THE WORLD}

The number of participants at the XIV International Congress of Applied Psychology in Copenhagen was 1,413 , it is reported. Asia (Japan 8 , Israel 4, Thailand 1), Europe (Denmark 434, Sweden 102, U.K. 92, Germany 89, France 70, Holland 59 , Norway 35 , Italy 29 , Finland 27 , Belgium 26 , Switzerland 27, Austria 9, Hungary 5, Czechoslovakia 5, Poland 4, Rumania 5, U.S.S.R. 3, Yugo- slavia 4, Iceland 3, Ireland 2, Spain 1, Portugal 1), America (U.S. A. 344, Brazil 7, Argentina 3), Australia (Australia 2, New Zealand 1), Africa (Congo 1).

The Institution for Phonetic Sciences, Kyoto University, published No. 1 of Studia Phonologica, written in Japanese with English abstract. 